\title{
DAX-1, as an androgen-target gene, inhibits aromatase expression: a novel mechanism blocking estrogen- dependent breast cancer cell proliferation
}

\author{
M Lanzino ${ }^{1,2}$, P Maris ${ }^{1}$, R Sirianni ${ }^{1}$, I Barone $e^{1,2}$, I Casaburi ${ }^{1}$, A Chimento ${ }^{1}$, C Giordano ${ }^{2}$, C Morelli ${ }^{1}$, D Sisci ${ }^{1,2}$, P Rizza ${ }^{1}$, \\ D Bonofiglio ${ }^{1,2}, S$ Catalano ${ }^{1,2}$ and S Andò $\dot{o}^{*, 1,2}$
}

Sexual hormones, estrogens and androgens, determine biological response in a tissue- and gender-specific manner and have a pivotal role in endocrine-mediated tumorigenesis. In situ estrogen production by aromatase is a critical determinant for breast cancer growth and progression. On the contrary, clinical and in vitro studies indicate that androgens have a protective role in mammary carcinogenesis. Here, we demonstrated, in hormone-dependent breast cancer cells, the existence of a functional interplay between the androgen receptor (AR), the orphan nuclear receptor DAX-1 and the aromatase enzyme involved in the inhibition of the estrogen-dependent breast cancer cell proliferation exerted by androgen signaling. Indeed, our results revealed, in MCF-7 cells, that ligand-activated AR induces the expression of the orphan nuclear receptor DAX-1 by direct binding to a newly identified androgen-response-element within the DAX-1 proximal promoter. In turn, androgen-induced DAX-1 is recruited, in association with the corepressor $\mathrm{N}-\mathrm{CoR}$, within the SF-1/LRH-1 containing region of the aromatase promoter, thereby repressing aromatase expression and activity. In elucidating a novel mechanism by which androgens, through DAX-1, inhibit aromatase expression in breast cancer cell lines, these findings reinforce the theory of androgen- opposing estrogen-action, opening new avenues for therapeutic intervention in estrogen-dependent breast tumors.

Cell Death and Disease (2013) 4, e724; doi:10.1038/cddis.2013.235; published online 11 July 2013

Subject Category: Cancer

Estrogens influence the pathological processes of several hormone-dependent cancers, such as breast, endometrial, ovarian and testicular tumors. ${ }^{1,2}$ In the breast, estrogen signaling has a critical role in cell proliferation and tumor development, ${ }^{3}$ and estrogen receptor $\alpha(E R \alpha)$-positive tumors comprise $\sim 70 \%$ of annually diagnosed breast cancer cases. ${ }^{4}$ Importantly, in addition to the ovarian estrogen supply, biologically active estrogens, especially $17-\beta$-estradiol $\left(\mathrm{E}_{2}\right)$, are locally produced, through conversion from circulating adrenal androgens by the aromatase enzyme, in an intracrine mechanism within the breast cancer tissues. ${ }^{5}$ Consequently, substantial efforts have been devoted to explore ways to block estrogen activity in breast cancer cells, underpinning the widespread use of antiestrogens and aromatase inhibitors in the adjuvant treatment of breast cancer. ${ }^{6}$ Nevertheless, some patients fail to respond to current targeted therapies because of the absence of the necessary molecular tumor target or the onset of resistance to therapy. It is now turning out that a multi-faceted treatment is needed for therapy to be successful, rendering it necessary to utilize as many therapeutic targets as possible.
The balance between $\mathrm{ER} \alpha$ and androgen receptor (AR) signaling has been proposed as a critical determinant of growth in the normal and malignant mammary epithelium, supporting the prevalent theory of androgens opposing estrogens in the mammary gland. A significant number of primary well-differentiated breast tumors expresses $A R,{ }^{7}$ whose presence and functional activity appear to be related to positive prognostic factors, including ER-positivity, smaller tumor size, low tumor grade, improved response to hormone therapy and longer patient survival. ${ }^{8-10}$ Interestingly, several events involved in breast cancer genesis or progression have been shown to alter AR expression or function, conferring a growth advantage to cancer cells. Indeed, a trend towards a loss of AR has been shown in BRCA1-mutated breast tumors $^{11}$ as well as in HER2-positive breast cancers, ${ }^{12}$ generally associated with a worse outcome. These findings are consistent with cell-based assays, indicating that, in ER/AR-positive breast tumor cell lines, AR activation by the agonist dihydrotestosterone decreases $\mathrm{ER} \alpha$ transcriptional activity $^{10,13}$ and inhibits basal as well as estrogen-dependent

\footnotetext{
${ }^{1}$ Department of Pharmacy and Health and Nutritional Sciences, University of Calabria, Arcavacata di Rende (CS), Italy and ${ }^{2}$ Centro Sanitario, University of Calabria, Arcavacata di Rende (CS), Italy

*Corresponding author: S Andò, Full Professor General Pathology, Department of Pharmacy, Nutritional and Health Sciences, University of Calabria, Arcavacata di Rende (CS) 87036, Italy. Tel: + 39 0984496201; Fax: + 39 0984496203; E-mail: sebastiano.ando@ unical.it

Keywords: breast cancer; androgen receptor; DAX-1; aromatase

Abbreviations: AR, Androgen Receptor; Mb, Mibolerone; DHT, Dihydrotestosterone; OH-Fl, Hydroxyflutamide; AHC, Dosage-sensitive sex reversal, Adrenal Hypoplasia Congenita; DAX-1, critical region on chromosome X, gene 1; NROB1 N-CoR; SF-1, Steroidogenic Factor-1; LRH-1, Liver Receptor Homolog-1; ER $\alpha$, Estrogen Receptor-alpha; $E_{2}, 17-\beta$ estradiol; BRCA1, Breast Cancer Type 1 susceptibility protein; HER2, Human Epidermal Growth Factor Receptor 2; ARE, Androgen Response Element; shRNA, Short Hairpin RNA; DAPA, DNA Affinity Precipitation Assay; EMSA, Electrophoretic Mobility Shift Assay; ChIP, Chromatin ImmunoPrecipitation

Received 08.1.13; revised 07.5.13; accepted 23.5.13; Edited by G Melino
} 
cell proliferation. ${ }^{14-16}$ These effects may occur via a decrease in cyclin D1 gene expression through an AR-mediated mechanism involving the participation of the orphan nuclear receptor DAX-1 (dosage-sensitive sex reversal, adrenal hypoplasia congenita $(\mathrm{AHC})$ critical region on chromosome $X$, gene 1 ; NROB1). ${ }^{16}$

DAX-1 is an unusual orphan member of the nuclear receptor superfamily, lacking the classical zinc-finger DNAbinding domain, ${ }^{17,18}$ that instead of directly binding to regulatory DNA sites, controls transcription mainly as a corepressor by associating with nuclear receptors (e.g., AR, $\mathrm{ER}$ ), or other transcription factors (e.g., steroidogenic factor-1, SF-1 or Liver Receptor Homolog-1, LRH-1). DAX-1 has a restricted expression pattern to tissues directly involved in steroid hormone production and reproductive function, such as adrenal cortex, Leydig and Sertoli cells in the testis, and theca and granulosa cells in the ovary. ${ }^{19-21}$ Within these tissues, DAX-1 acts as a global anti-steroidogenic factor by working in pair with SF-1/LRH-1 and repressing the expression of multiple enzymes involved in the steroidogenic pathway including aromatase. ${ }^{19,21-24}$ DAX-1 expression has also been reported in several types of cancers. In adrenocortical tumors, DAX-1 presence is inversely correlated to the level of steroid production. ${ }^{25} \mathrm{DAX}-1$ expression in breast, ${ }^{26-28}$ ovarian, $^{29}$ endometrial $^{30}$ and prostate cancers ${ }^{31}$ has been additionally described, although the mode of its regulation is not narrowly investigated.

Here, we identify a novel AR-mediated mechanism controlling the expression of DAX-1 and consequently of aromatase. On the basis of our findings, ligand-activated AR may negatively regulate in situ estrogen production by activating $D A X-1$ gene transcription in estrogen-related breast cancer cells, providing new clues for a better comprehension of the mechanisms underlying the inhibitory role exerted by androgens in estrogen-dependent cancer cell proliferation in the breast.

\section{Results}

Ligand-activated AR increases DAX-1 expression in MCF-7 cells. Our first aim was to investigate the ability of androgen to modulate the expression of the orphan nuclear receptor DAX-1. In the present study, experiments were carried out using the synthetic AR agonist Mibolerone $(\mathrm{Mb})$ to minimize the metabolic conversion of androgen to estrogenic compounds by cells in culture. As expected, Mb appeared to be as effective as dihydrotestosterone $(\mathrm{DHT})^{14-16}$ in inhibiting MCF-7 breast cancer cell proliferation. Indeed, Mb administration was able to inhibit cell proliferation in a dose-dependent manner (Figure 1a) and to induce apoptosis as indicated by Tunel assay, showing a marked increase in the number of apoptotic nuclei upon 6 days of $\mathrm{Mb}$ treatment (Figures $1 \mathrm{~b}$ and $\mathrm{c}$ ). Then, we evaluated the effects of $\mathrm{Mb}$ administration on DAX-1 levels and cellular compartmentalization in human MCF-7 breast cancer cell line. As shown in Figure $2 \mathrm{a}$, treatment with $\mathrm{Mb}$ increased both DAX-1 mRNA and protein cellular levels. Immunofluorescence and immunoblotting analysis (Figures $2 b$ and $c$ ) revealed that DAX-1 protein localizes in both the cytoplasm and nucleus, although a stronger immunoreactivity was observed in the nuclear compartment. $\mathrm{Mb}$ administration induced a significant increase in DAX-1 nuclear abundance. The involvement of activated $A R$ in the androgen-dependent regulation of DAX-1 was ascertained by using a shRNA interfering approach able to cause a reduction in AR expression in MCF-7 cells that can be particularly appreciated in the nuclear compartment (Figure 2c).

Ligand-activated $A R$ induces $D A X-1$ gene promoter activity. To determine whether the human $D A X-1$ gene is a target of ligand-activated $\mathrm{AR}$, transient transfection experiments were performed on MCF-7 cells (Figure 3a). $\mathrm{Mb}$ administration induced a significant enhancement of DAX-1 promoter activity. This effect was reversed by the addition of the AR antagonist, Hydroxyflutamide $(\mathrm{OH}-\mathrm{Fl})$, indicating that it was mediated by $A R$ activation. Similar results were observed following treatment with the AR natural ligand DHT (data non shown). To confirm the involvement of ligand-activated $A R$ in the modulation of DAX-1 gene expression, we tested the ability of ectopic AR to trigger the activation of $D A X-1$ promoter in AR-negative HEK293 cells (Figure $3 b$ ). In the absence of exogenous AR expression, $D A X-1$ promoter activity was not influenced by $\mathrm{Mb}$ administration. Conversely, in the presence of ectopic $\mathrm{AR}$, activation of $D A X-1$ transcriptional activity was observed in response to $\mathrm{Mb}$ treatment. To explore whether the activation of $D A X-1$ promoter was dependent on the $A R$ DNA-binding and transactivating properties, HEK293 cells were transfected with increasing concentration of an expression plasmid encoding an AR carrying a mutation (AR $\left.\mathrm{A}_{\mathrm{Cys} 574 \rightarrow \mathrm{Arg}}\right)$ in the DNA-binding domain. ${ }^{32}$ In these experimental conditions, no changes in DAX-1 promoter activity were observed (Figure $3 b$ ), indicating that DAX-1 promoter can be considered a target of $\mathrm{AR}$ and strongly suggesting the existence, within the DAX-1 promoter, of putative androgen responsive region(s).

Identification of a functional androgen responsive element (ARE) within the DAX-1 promoter. Sequence analysis revealed that the $D A X-1$ promoter contains a putative androgen response element (ARE) located at position $-1074 \mathrm{bp}$ upstream of the translation initiation codon, which is homologous to the AREs found in the promoters of several androgen responsive genes ${ }^{33}$ (Figure 4a).

To assess whether this putative ARE sequence might be crucially involved in DAX-1 promoter responsiveness to androgens, site-directed mutagenesis was used to alter it by introducing nucleotide substitutions. The mutant promoter mDAX-1-Luc was then assayed in functional analysis in parallel with the unmutated one. Disruption of the putative ARE consensus site resulted in a significant loss of the upregulatory effect of $\mathrm{Mb}$ on $\mathrm{DAX}-1$ promoter activity (Figure $4 \mathrm{~b}$ ), addressing the ARE motif as a critical sequence in mediating $\mathrm{DAX}-1$ promoter activation upon $\mathrm{Mb}$ exposure.

To further investigate the functional importance of the identified ARE sequence (DAX-1-ARE), we tested the hypothesis that AR could effectively bind to it. To this aim, DAPA was performed by using a double-stranded oligonucleotide containing the core ARE sequence (Figure 5a). 

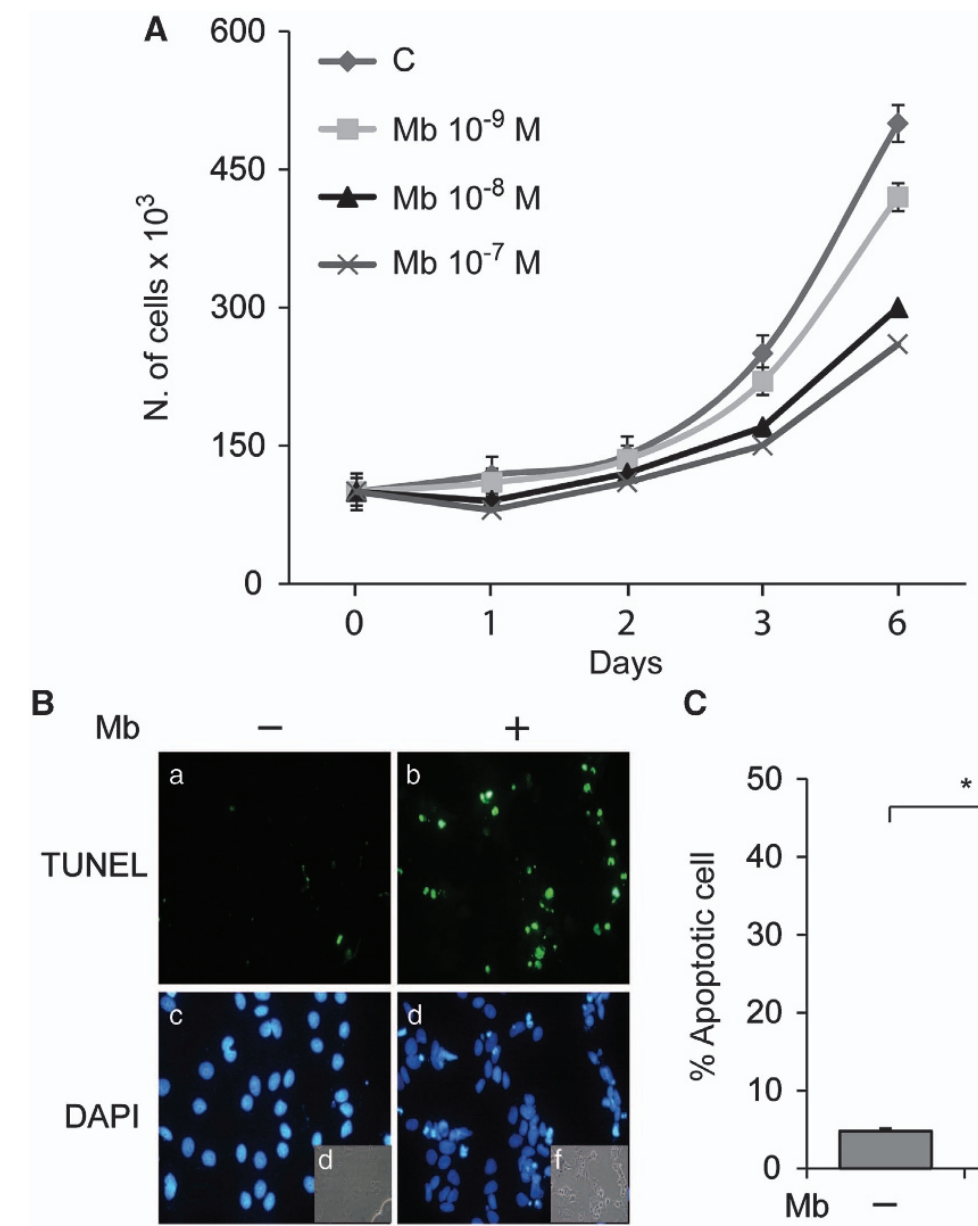

C

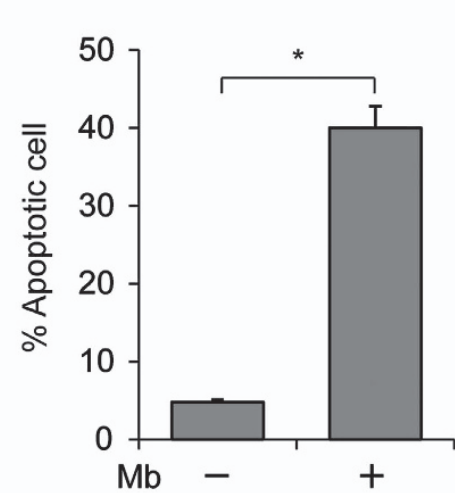

Figure 1 Long-term Mb administration inhibits MCF-7 cell proliferation and induces apoptosis. (A) Cell counting by trypan blue exclusion test in cells treated as indicated ( 6 days). (B) Cells treated with $10^{-8} \mathrm{M}$ Mb for 6 days were subjected to TUNEL nuclear staining $(\mathbf{a}, \mathbf{b})$ and viewed by a fluorescent microscope. DAPI staining for nuclei detection (c, d); bright field $(\mathbf{e}, \mathbf{f})$. Apoptotic cells were photographed at $\times 10$ magnification and then counted using Image $\mathrm{J}$ software. (C) Histograms represent the apoptotic index \% apoptotic cells/total cell number in the field). ${ }^{*} P<0.01$

Endogenous AR was found associated with the putative consensus oligonucleotide following $\mathrm{Mb}$ administration, only. A mutant oligonucleotide abolished AR binding, indicating that the in vitro DNA-AR binding is sequence specific. Association between $A R$ and the ARE-containing oligonucleotide was also detected upon DHT administration (data not shown). Additionally, EMSA was also performed using the identified ARE motif as a DNA probe (Figure $5 b$ ). Factors present in MCF-7 nuclear extracts and ARE probe formed a protein-DNA complex (lane1), which was strongly enhanced upon $\mathrm{Mb}$ treatment (lane2). The specificity of the protein-DNA association was demonstrated by its disappearance in the presence of 100 -fold molar excess of unlabeled probe as competitor (lane3) or a mutated labeled probe (lane4). Coadministration of the anti-androgen Casodex markedly decreased the Mbinduced DNA-binding complex indicating the involvement of the AR (lane5). Moreover, the DNA-protein complex formation was immunodepleted in the presence of a specific anti-AR antibody, indicating that this antibody recognizes AR epitopes that interact or interfere with $A R$ association to $A R E$ (lane6). ChIP analysis was then used to determine whether endogenous $A R$ localizes to the native $D A X-1$ promoter in MCF-7 breast cancer cells (Figure 5c, upper panel). AR occupancy of the DAX-1-ARE-containing region of DAX-1 promoter was induced in a ligand-dependent manner, as $A R$ recruitment was enhanced by $M b$ administration. Moreover, the dynamic of RNA Pol II recruitment onto the $D A X-1$ promoter further evidenced the ability of $\mathrm{AR}$ to transactivate the $D A X-1$ gene. These results were also reproduced in the $A R / E R-p o s i t i v e ~ T 47 D$ breast cancer cell line (Figure 5c, lower panel).

Overall, these findings clearly demonstrated that ligandactivated AR can bind to the ARE motif within the DAX-1 proximal promoter in human breast cancer cells.

DAX-1 overexpression induces an apoptotic switch in breast cancer cells. To support the biological significance of the upregulation of DAX-1 levels induced by androgens, DAX-1 was ectopically overexpressed in MCF-7 cells to test whether DAX-1-mediated signals might influence cell proliferation. Interestingly, increased DAX-1 cellular content affected MCF-7 population number by reducing cell 
A

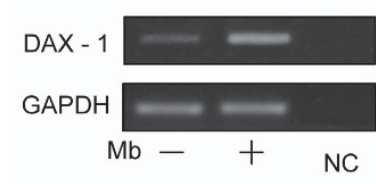

C
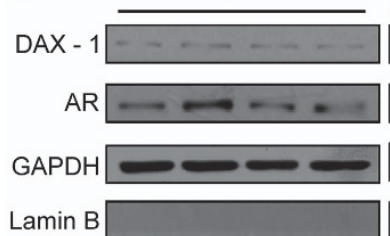

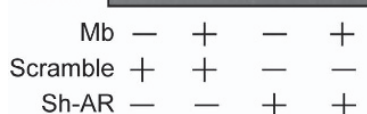

DAX - 1

$\beta$-Actin

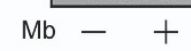

Nucleus
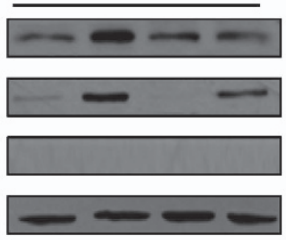

$-+-+$

\begin{tabular}{l}
+ \\
$+\quad+-1$ \\
\hline
\end{tabular}
B $\mathrm{Mb}$
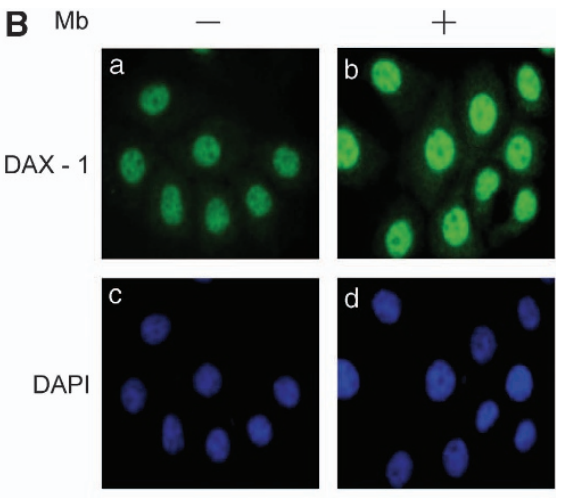

BF
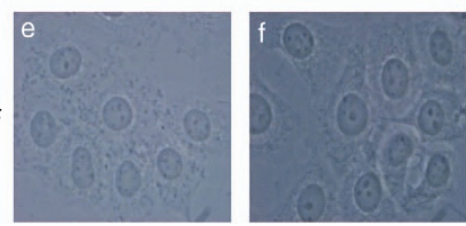

Figure 2 Ligand-activated AR modulates DAX-1 expression in MCF-7 cells. (A) RT-PCR and immunoblotting of DAX-1 in cells treated with $10-8$ M Mb for $24 \mathrm{~h}$. GAPDH internal standard; NC, negative control. $\beta$-Actin, loading control. (B) Immunofluorescence of DAX-1 (a, b) and DAPI (c, d) in cells treated with $10^{-8} \mathrm{M} \mathrm{Mb}$ for $24 \mathrm{~h}$. BF, bright field (e,f). (C) Immunoblotting of DAX-1 and AR from cells transfected with $0.5 \mu \mathrm{g} \mathrm{pSiAR}$ (sh-AR) or $0.5 \mu \mathrm{g}$ pSiCon (Scramble) and treated with $10^{-8} \mathrm{M}$ Mb for $24 \mathrm{~h}$. GAPDH and Lamin-B, control of loading and purity of lysate fractions. Results are representative of three independent experiments

a

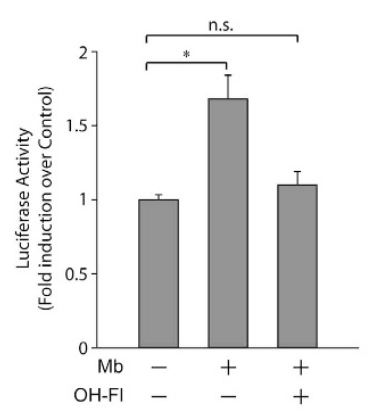

b

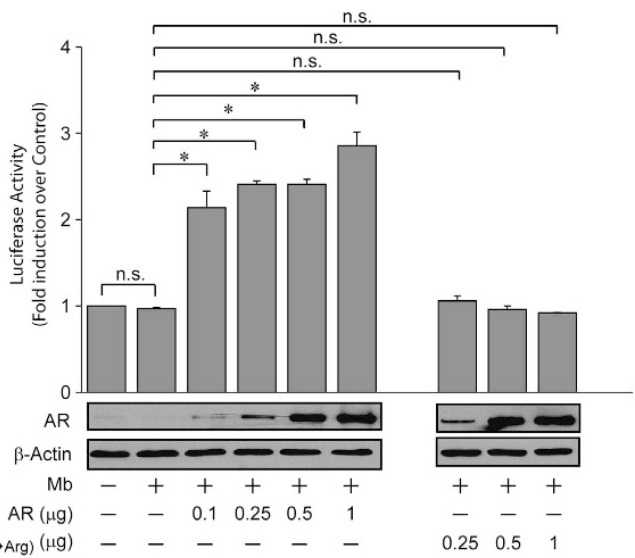

Figure 3 AR activates DAX-1 gene promoter. (a) MCF-7 cells, transfected with DAX-1-Luc-plasmid, were treated with $10^{-8} \mathrm{M} \mathrm{Mb}$ and/or $10^{-6} \mathrm{M} \mathrm{OH}-\mathrm{Fl}$, (24 h). (b) HEK293 cells, co-transfected with DAX-1-Luc-plasmid plus increasing amounts of AR or AR Immunoblotting for $\mathrm{AR}$ and $\beta$-actin (loading control) expression. ${ }^{*} P<0.01$; n.s., nonsignificant

a

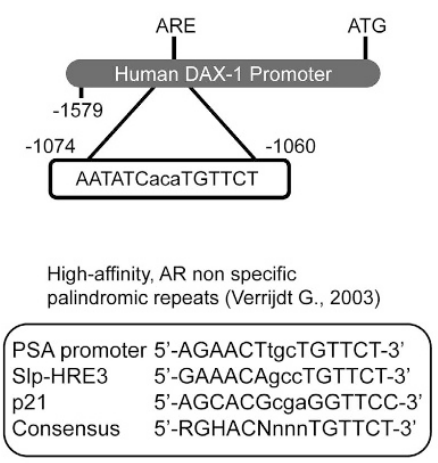

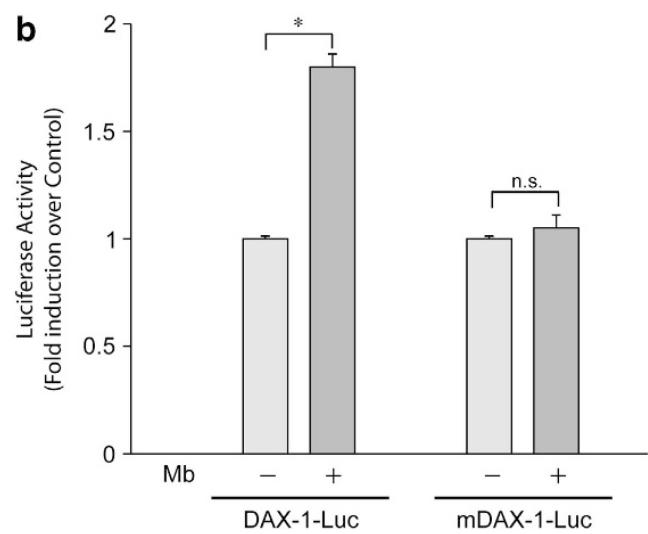

Figure 4 DAX-1 promoter contains a putative androgen response element (ARE). (a) Schematic representation of ARE site and list of known ARE sequences. (b) MCF-7 cells, transfected with wild type DAX-1-Luc-plasmid or ARE-mutant DAX-1-Luc-plasmid (mDAX-1-Luc) were treated with $10^{-8} \mathrm{M}$ Mb for $24 \mathrm{~h} .{ }^{*} P<0.01$; n.s., nonsignificant 
a
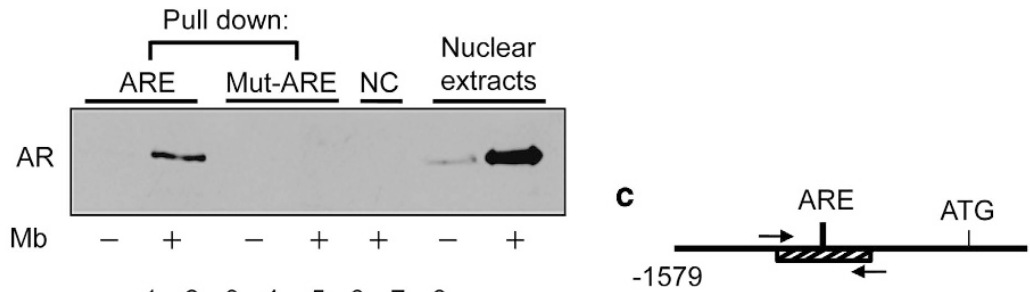

b

$\begin{array}{llllllll}1 & 2 & 3 & 4 & 5 & 6 & 7 & 8\end{array}$
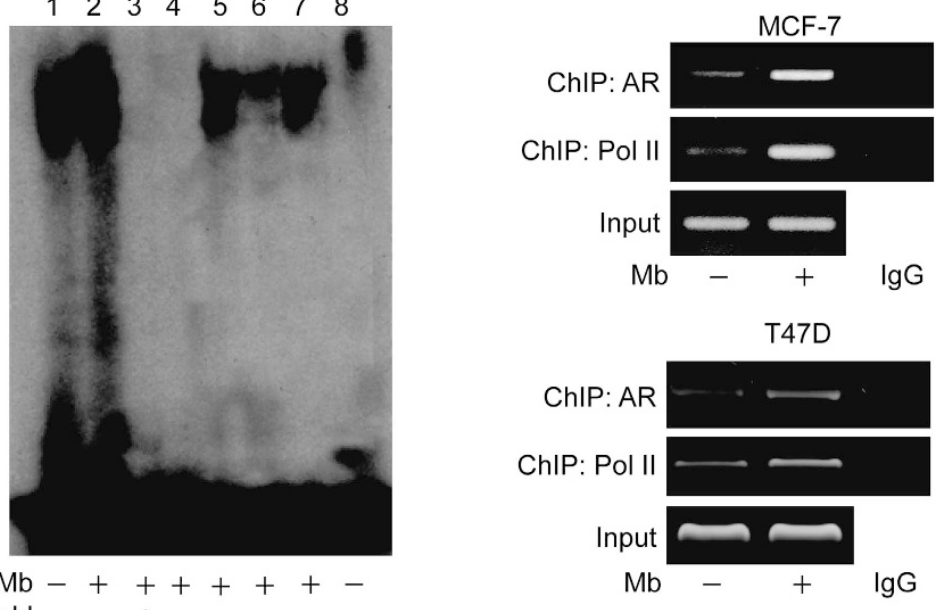

$\mathrm{Mb}-++++++-$

Cold --+----

Mut Probe ARE - - - + - - -

Casodex - - - + - -

$\mathrm{Ab}$ anti AR -----+-

$\lg \mathrm{Ig}----+$

Probe +++-++++

Figure 5 Ligand-activated AR binds to an ARE site within DAX-1 promoter. (a) DAPA on nuclear extracts from MCF-7 cells treated with $10^{-8} \mathrm{M}$ Mb for $2 \mathrm{~h}$. Wild-type (ARE) or mutated (mut-ARE) biotinylated oligonucleotide were used. Unbound fraction, negative control (NC); nuclear extracts, positive control. (b) EMSA on nuclear extracts from MCF-7 cells untreated (lane 1) or treated with $10^{-8} \mathrm{M}$ Mb for $2 \mathrm{~h}$ (lane 2-7). 100-fold molar excess of unlabeled probe (lane 3); labeled mutated probe (lane 4); addition of $10^{-5} \mathrm{M}$ Casodex (lane 5); pre-incubation with anti-AR antibody (lane 6) or IgG (lane 7); probe alone (lane 8). (c) Sheared chromatin from MCF-7 or T47D cells treated with $10^{-8} \mathrm{M} \mathrm{Mb}$ for $2 \mathrm{~h}$ was precipitated using anti-AR or anti-RNA Pol II antibodies. IgG, control samples. DNA input, loading control. Results are representative of three independent experiments

proliferation rate (Figure 6a). This phenomenon was associated to apoptotic cell death as indicated, at day 6 , by increased PARP cleavage, caspase 9 activation (Figure 6b), as well as enhanced $\mathrm{Bad} / \mathrm{Bcl}-2$ ratio (Figures $6 \mathrm{~b}$ and $\mathrm{c}$ ) and enhanced presence of pyknotic nuclei (Figures $6 \mathrm{~d}$ and e).

Androgen treatment inhibits aromatase expression through a DAX-1-mediated mechanism in MCF-7 cells. To disclose the functional relationship between the androgen-dependent upregulation of DAX-1 expression and breast cancer cell proliferation, we explored the possibility that it might affect in situ estrogen production as the aromatase gene has been shown to represent a physiologic target gene for DAX-1. ${ }^{24}$ To this aim, the expression of P450aromatase upon androgen treatment was examined in MCF-7 cells. Mb administration decreased the cellular content of the enzyme at both mRNA and protein levels (Figure 7a). The participation of DAX-1 in the androgen-dependent modulation of aromatase expression was demonstrated using a siRNA technology (Supplementary Figure S1). Indeed, DAX-1 gene silencing abrogated the down-regulatory effect induced by $\mathrm{Mb}$ administration on aromatase mRNA and protein levels (Figure 7b).
Previous studies demonstrated that DAX-1 is able to repress SF-1/LRH-1-mediated transactivation of several steroidogenic genes, including P450aromatase, by acting as an adaptor molecule and recruiting the steroid receptor corepressor $\mathrm{N}-\mathrm{CoR} .{ }^{22-24}$ Accordingly, the occurrence of a similar mechanism in our experimental system upon androgen treatment was investigated. The ability of $\mathrm{Mb}$ to regulate aromatase gene transcriptional activity through the PII-1.3 proximal promoter was evaluated first by transient transfection-based reporter gene assays in MCF-7 cells (Figure 7c). $\mathrm{Mb}$ administration reduced promoter transcriptional activity. On the contrary, no significant changes were observed in cells transfected with a PII-1.3 reporter plasmid bearing a SF-1/ $\mathrm{LRH}-1$-mutated binding site, underlying the importance of this motif in the regulation of aromatase expression by androgens in MCF-7 breast cancer cells. Moreover, ChIP assay revealed that $\mathrm{Mb}$ administration significantly induced $\mathrm{DAX}-1$ recruitment within the SF-1/LRH-1-containing region of the PII-1.3 aromatase proximal promoter (Figure $7 d$ ). According with the repressive role of $\mathrm{DAX}-1$ in the transcriptional activity of the aromatase gene, its increased recruitment is paralleled by an enhanced occupancy of the same promoter region by the nuclear receptor co-repressor N-CoR (Figure 7d). 
A

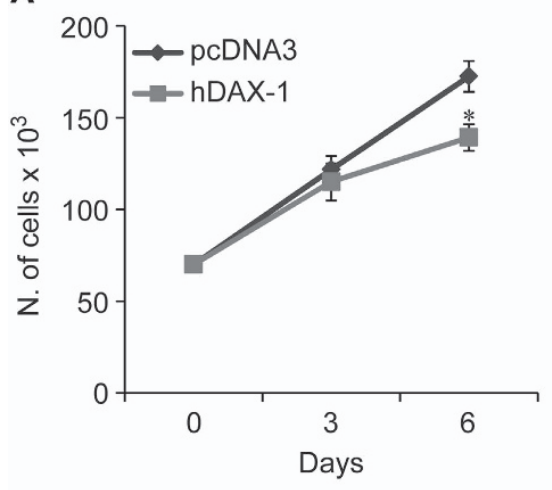

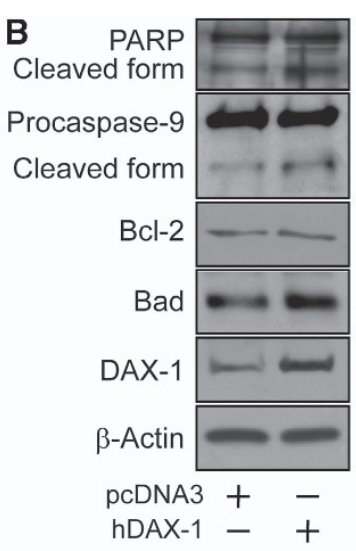

C

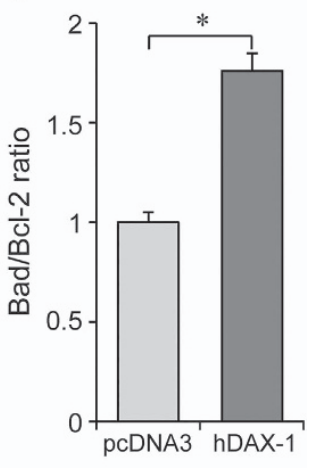

D

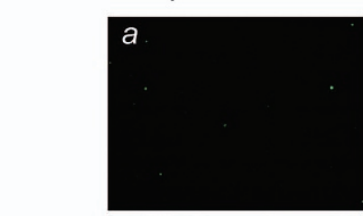

DAPI
hDAX-1

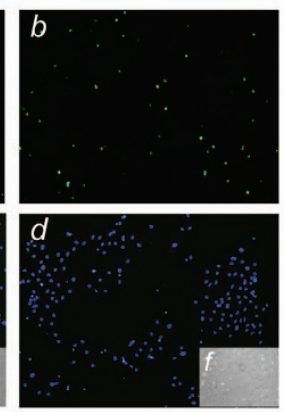

E

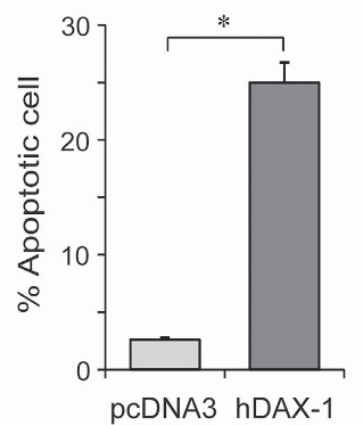

Figure 6 DAX-1 overexpression decreases proliferation and induces apoptosis in MCF-7 cells. (A) Cells were transfected with control plasmid (pcDNA3) or a human DAX1 expressing plasmid (hDAX-1). Transfection was renewed every 2 days and cells were counted on day 6 using trypan blue exclusion test. ${ }^{\star} P<0.05$. (B) Immunoblotting analysis for PARP cleavage, Caspase 9, Bcl-2, Bad and DAX-1 expression in total extracts from cells transfected as above. $\beta$-Actin, loading control. (C) Histograms represent the ratio between Bad and bcl-2 band intensities (from B) evaluated as optical density arbitrary units and expressed as fold induction over control. ${ }^{*} P<0.01$. (D) MCF-7 cells, transfected as above, were subjected to TUNEL nuclear staining $(\mathbf{a}, \mathbf{b})$ and viewed by a fluorescent microscope. DAPI staining for nuclei detection (c, d); bright field $(\mathbf{e}, \mathbf{f})$. Apoptotic cells were photographed at $\times 10$ magnification and then counted using Image $\mathrm{J}$ software. (E) Histograms represent the apoptotic index (\% apoptotic cells/total cell number in the field). ${ }^{*} P<0.01$

\section{Discussion}

In human hormone-sensitive neoplasms, such as Leydig cell and breast tumors, estrogens can be de novo produced, ${ }^{2,34-36}$ indicating an essential role for the androgen local aromatization by P450aromatase. Accordingly, aromatase inhibitors are currently established as good pharmaceutical tools for the treatment of ER-positive breast cancers. However, resistance to this therapy still remains to be solved by other methods of suppression of intra-tumoral estrogen production. ${ }^{5}$ Hence, analysis of the modulation of intratumoral aromatase appears to be crucial not only for a better comprehension of the development and the biological behavior of estrogen-dependent breast carcinomas, but also in the clinical management of cancer patients. In the present study, we demonstrate a novel androgen receptor-mediated mechanism leading to the activation of the DAX-1 gene transcription, which, in turn, negatively affects on aromatase expression and activity in hormone-dependent MCF-7 breast cancer cells.

Orphan nuclear receptor DAX-1 has key roles in the development and the maintenance of reproductive function and hormone biosynthesis in mammals. ${ }^{19,37-39}$ However, DAX-1 regulation and functions in sex steroid-dependent neoplasms, in which in situ steroid hormone production and metabolism hold a crucial role, need to be further clarified. In node-negative breast cancer, DAX-1 expression correlates with positivity for $E R$ and $A R^{26,27}$ smaller tumor size and excellent survival. ${ }^{40}$ Nevertheless, although several studies established DAX-1 as a crucial regulator of steroidogenesis, the precise molecular mechanism behind DAX-1 transcriptional control and/or its relationship to aromatase expression and activity in breast cancer cells still remains largely unknown. Our study, for the first time, identifies DAX-1 as an androgen-responsive gene, whose expression is positively modulated by the ligand-activated AR. Indeed, in MCF-7 breast cancer cells, DAX-1 expression is upregulated by administration of the AR agonist Mibolerone as evidenced by enhancement of its mRNA and protein levels and increase in its promoter activity. These results are supported by previous observations in rat sertoli cells, indicating that DAX-1 expression is regulated during spermatogenesis and peaks during the androgen-sensitive phase of the spermatogenic cycle. ${ }^{41}$ Activated AR has a fundamental role in the upregulation of DAX-1 cellular levels as in AR-negative HEK293 cells, induction of DAX-1 promoter activity can be evidenced only in the presence of exogenous AR expression and strictly depends on the integrity of the AR DNA-binding domain, suggesting the existence, in the DAX-1 promoter, of putative androgen responsive region(s), which mediate 
a

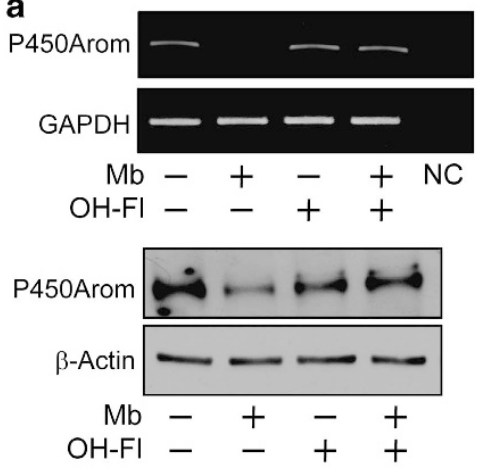

b

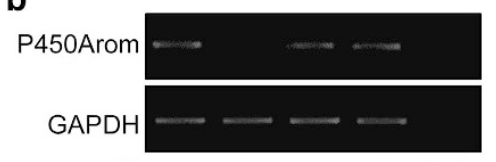

$\mathrm{Mb}-+-+\mathrm{NC}$

si-scramble ++-

si-DAX-1 - -++

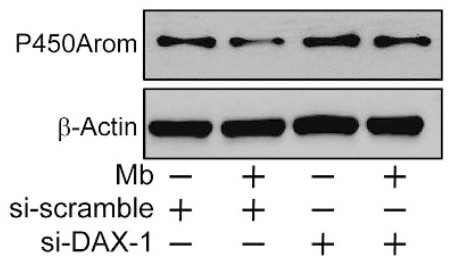

C
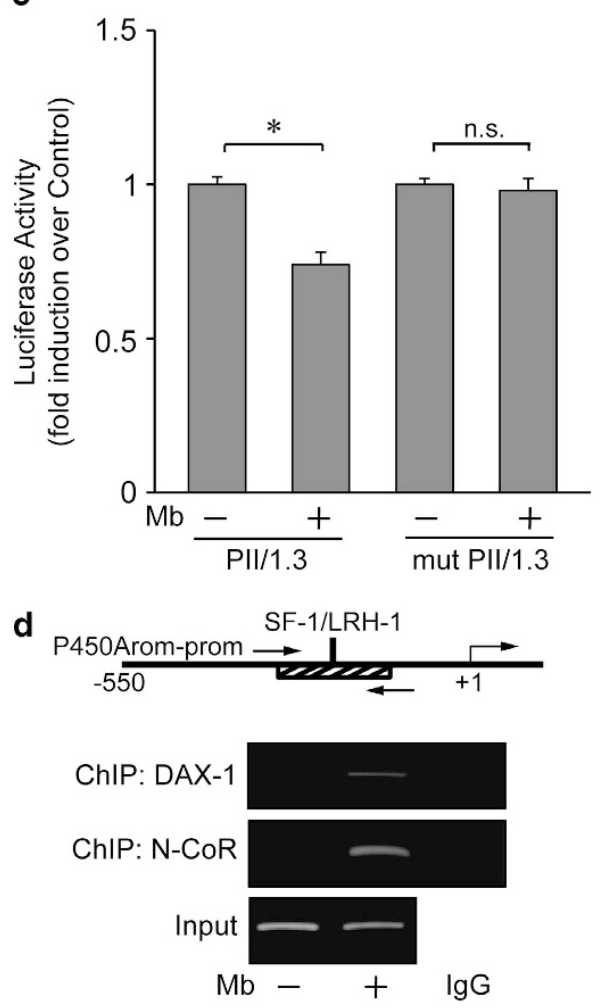

Figure 7 Mibolerone through DAX-1 inhibits P450aromatase expression in MCF-7 cells. (a) RT-PCR and immunoblotting of P450aromatase expression in cells treated with $10^{-8} \mathrm{M} \mathrm{Mb}$ and/or $10^{-6} \mathrm{M} \mathrm{OH}$-FI for 48h. GAPDH, internal standard; NC, negative control. $\beta$-Actin, loading control. (b) RT-PCR and immunoblotting of P450aromatase in cells transfected with siDAX-1 or scrambled control siRNA (si-scramble) and treated with $10^{-8} \mathrm{M} \mathrm{Mb}$ for $48 \mathrm{~h}$. GAPDH, internal standard; NC, negative control. $\beta$-Actin, loading control. (c) Cells, transfected with PII/1.3- or SF-1/LRH-1-mutant PII/1.3- (mut PII/1.3) aromatase promoter plasmids, were treated with $10^{-8} \mathrm{M}$ Mb for $24 \mathrm{~h} .{ }^{*} P<0.05$; n.s., nonsignificant. (d) Sheared chromatin from MCF-7 cells treated with $10^{-8} \mathrm{M} \mathrm{Mb}$ for $2 \mathrm{~h}$ was precipitated using anti-DAX-1 or anti-N-CoR antibodies. IgG, control samples. DNA input, loading control. Results are representative of three independent experiments

androgen response. In the current study, we identified an androgen response element (DAX-1-ARE: 5'-AATATCa caTGTTCT-3') located between $-1074 \mathrm{bp}$ and $-1060 \mathrm{bp}$ upstream of the $D A X-1$ gene translation initiation codon, which displays the conventional inverted repeats of the $5^{\prime}$-TGTTCT-3' monomer-binding element found in the promoters of several androgen-responsive genes. ${ }^{33}$ This DAX-1-ARE is functional, as demonstrated by transactivation studies, and capable to bind efficiently the $A R$ in a ligand-dependent manner. Furthermore, the in vivo interaction between $A R$ and the DAX-1 promoter is supported by ChIP analysis showing that AR occupancy of the DAX-1-ARE-containing promoter region is concomitant with an increase in RNA Polymerase II recruitment, consistent with the enhanced $D A X-1$ transcriptional activity. Thus, activated AR may directly modulate the expression of DAX-1, and hence it may lie upstream of DAX-1 in a regulatory cascade directing steroid hormones biosynthesis in various steroidogenic tissues. Indeed, DAX-1 has significant roles in the regulation of steroidogenesis through a variety of mechanisms of transcriptional repression and interaction with many other factors. Interestingly, studies on DAX-1 deficient mice, displaying an increased aromatase expression, indicate that P450aromatase is a physiological target for DAX-1 in Leydig cells. ${ }^{24}$ Consistent with these observations, we found that androgen-dependent upregulation of DAX-1 significantly affects on aromatase content in breast cancer cells as demonstrated by the evidence that silencing $D A X-1$ gene completely reversed the downregulatory effect exerted by androgens on aromatase expression. Transcriptional control by DAX-1 occurs through its recruitment on the SF-1/LRH-1-binding site within the $\mathrm{PII} / 1.3$ aromatase proximal promoter in association with the transcriptional corepressor $\mathrm{N}-\mathrm{CoR}$, leading to inhibition of aromatase gene transcription. The importance of our results is highlighted by recent studies demonstrating that intratumoral DHT levels are positively associated with AR and 5- $\alpha$-reductase- 1 expression but inversely correlated with tumor size, Ki-67 and aromatase expression. ${ }^{42}$ Thus, in AR-positive breast carcinomas the use of aromatase inhibitors may be more effective by accumulation of the local DHT concentration, which, in turn, on acting in an autocrine short loop through DAX-1, might further contribute to reduce aromatase levels ${ }^{43}$ that are crucial in the maintenance of breast cancer cell growth. Besides, our findings show that DAX-1 levels represent an important limiting factor for MCF-7 cell proliferation. Indeed, DAX-1 overexpression per se is sufficient to reduce tumor cell number and is associated with a marked increase in the bad/ bcl-2 ratio switching to apoptotic cell death, presumably as a consequence of the estrogen withdrawal.

Therefore, the molecular mechanism described here might contribute to explain the negative influence of androgens on breast cancer cell proliferation, and well correlates with 


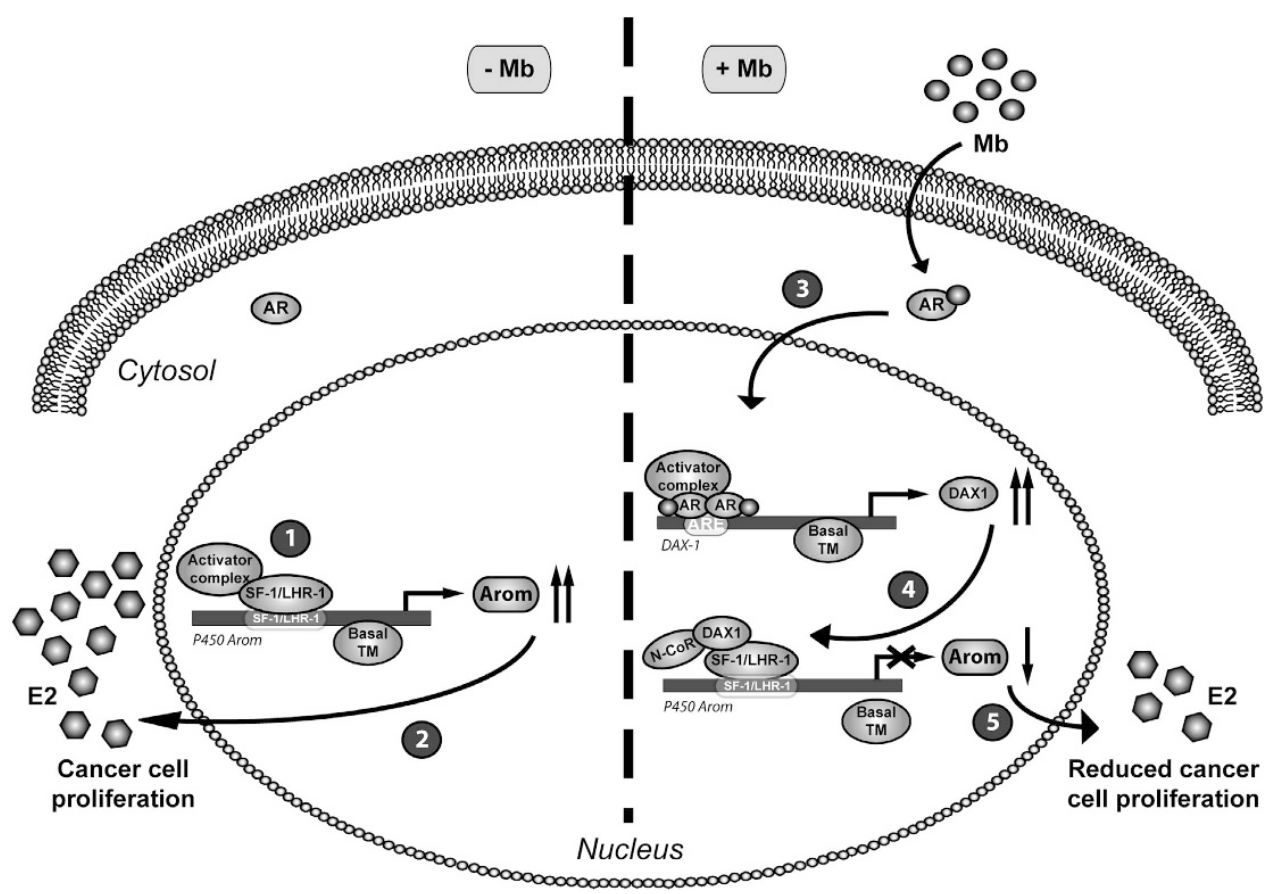

Figure 8 Proposed model for AR/DAX-1/Aromatase interplay in breast cancer cells. In the absence of androgens, P450aromatase (1) is regulated by several factors sustaining gene transcription and aromatase protein expression leading to (2) enhanced in situ estrogen $\left(\mathrm{E}_{2}\right)$ production responsible for cancer cell proliferation. In the presence of androgens, ligand-activated AR (3) binds to a specific ARE within DAX-1 promoter gene triggering its activation. In turn, increased DAX-1 protein (4) is recruited onto the P450aromatase promoter and represses SF-1/LHR-1-mediated transcription, reducing (5) locally produced estrogens thereby affecting cancer cell proliferation

previous findings in endometrial carcinoma, where loss or decrease in DAX-1 expression results in increased intratumoral steroid production and enhanced estrogen-dependent proliferation of cancer cells. ${ }^{30}$

Collectively, our model for AR-mediated repression of aromatase in estrogen-related breast cancers (Figure 8) proposes that ligand-activated AR directly binds to the DAX-1 promoter resulting in increased $D A X-1$ gene expression. In turn, DAX-1 represses aromatase through its recruitment within the SF-1/LRH-1-containing region of the aromatase promoter, causing reduced in situ estrogen production and inhibition of cancer cell proliferation.

Our study, for the first time, identifies DAX-1 as an androgen target gene and highlights the existence of a functional AR/DAX-1/aromatase interplay involved in the inhibition of the estrogen-dependent breast cancer cell proliferation induced by androgen signaling. Therefore, targeting $\mathrm{DAX}-1$ and $\mathrm{AR}$ pathways may represent an exploitable pharmacological tool in estrogen-related cancers, opening new avenues for therapeutic intervention.

\section{Materials and Methods \\ Reagents and antibodies. Mibolerone (Mb) from Perkin Elmer (Waltham, MA, USA); hydroxyflutamide (OH-FI) and 4',6-Diamidino-2-phenylindole (DAPI) from Sigma-Aldrich (St Louis, MO, USA); Bicalutamide (Casodex), bovine serum albumin (BSA) and AR (441 and 4i299), DAX-1 (K-17), Lamin-B (C-20), BAD (C- 7), BCl-2 (C-2), PARP-1/2 (H-250), RNA Polymerase II (N-20), N-COR (H-303), GAPDH (FL-335), $\beta$-actin (AC-15) antibodies from Santa Cruz Biotechnology (Dallas, TX, USA); Caspase-9 antibody (C9) from Cell Signaling (Danvers, MA, USA); human cytochrome P450aromatase antibody from Serotec (Oxford, UK).}

Cell cultures and animals. Authenticated human breast cancer cells MCF-7 (American Type Culture Collection-ATCC) and T47D (Interlab Cell Line
Collection), and human embryonic kidney cells HEK293 (ATCC) were acquired in 2010, stored according to the supplier's instructions and used within 4 months after frozen aliquots resuscitations. Every 4 months, cells were authenticated by single-tandem repeat analysis at our Sequencing Core; morphology, doubling times, estrogen sensitivity, and mycoplasma negativity were tested (MycoAlert, Lonza).

Before each experiment, cells were synchronized in phenol red-free serum free media (PRF-SFM) for $24 \mathrm{~h}$. All the experiments were performed in PRF-media containing $2.5 \%$ charcoal-treated fetal bovine serum (PRF-CT).

Cell proliferation assays. MTT assay and cell counting by trypan blue dye exclusion were performed as described. ${ }^{44}$

TUNEL assay. Apoptosis was determined as previously described ${ }^{45}$ by enzymatic labeling of DNA strand breaks by terminal deoxynucleotidyl transferasemediated deoxyuridine triphosphate nick-end labeling (TUNEL), using DeadEnd Fluorometric TUNEL System (Promega, Madison, WI, USA) according to the manufacturer's instructions. The slides were mounted using Fluoromount mounting medium (Sigma-Aldrich, Milan, Italy) and observed under a fluorescence microscope (Olympus BX51, Olympus Italia srl, Milan, Italy). Apoptotic cells were photographed at $\times 10$ magnification using ViewFinder ${ }^{\mathrm{TM}} 7.4 .3$ Software, through an Olympus camera system dp50 and then counted using Image J software $(\mathrm{NIH}$, Baltimore, MD, USA).

Plasmids, transfections and luciferase reporter assays. The following plasmids were used: pcDNA3-AR (AR) encoding human full-length $A R,{ }^{32}$ CMV-P881 (AR $($ Cys574 $\rightarrow$ Arg) $)$ encoding the full-length AR carrying a mutation in the DNA-binding domain (DBD; Cys-574 $\rightarrow$ Arg) ${ }^{32}$ the vector-based PSiAR plasmid encoding for small interfering RNA targeting the $5^{\prime}$-untranslated region of AR mRNA and scrambled control pSiCon; ${ }^{44}$ pcDNA3.1Zeo1 + vector encoding human DAX-1 (DAX-1); ${ }^{23}$ pGL3 vector containing human aromatase promoters II/ 1.3 (PII/1.3) (gift from Dr. E.R. Simpson and Dr. C.D. Clyne, Prince Henry's Institute of Medical Research, Australia).

A mutated SF-1/LHR-1 PI//1.3 plasmid was generated by deleting the SF-1/LHR-1 sequence in the human aromatase promoter PII/1.3 by PCR. The desired sequence deletions were confirmed by nucleotide sequence analysis, and the 
resulting plasmid encoding the mutated aromatase promoter II/1.3 was designated as mutPII/1.3 The luciferase expression vector DAX-1-Luc-promoter (DAX-1-Luc), encoding the DAX-1 promoter, was generated by PCR on genomic DNA. The following primer pairs were used to amplify a $1336 \mathrm{bp}$ fragment spanning from position $-1370 \mathrm{bp}$ to $-34 \mathrm{bp}$ from the ATG of the DAX-1 gene: $5^{\prime}$-GAG GAT GGG AGG GAG GGA AAA AGT-3' (forward) and 5'-AGG GCA GGG GAA AAG AGG AAA CAT-3' (reverse). PCR primer pairs were selected analyzing the $5^{\prime}$-flanking region of DAX-1 gene. The PCR product was purified and the $1336 \mathrm{bp}$ fragment was inserted in the pCR2.1 plasmid by using TOPO-TA cloning kit (Invitrogen, Milan, Italy) and sequenced. The fragment was cut with $\mathrm{Kpn} \mathrm{I}$ and Xho I and cloned into the pGL3 basic vector (Promega).

The human DAX-1 promoter plasmid bearing the androgen responsive element (ARE)-mutated site (mDAX-1-Luc) was generated by PCR using as template the DAX-Luc plasmid with the following primers: (mutation is shown as lowercase letters): forward 5'-ATA TGGTACC GCA GGA ACA GAA AAC CAA tttt CA CacaaT ATC ACT TGG AAG TGG GAG C-3' and reverse 5'-ATA TCT CGA GGT GGC TGC CTC CTG GGA CC-3'. The amplified DNA fragment was digested with Kpn I and Xho I and ligated into pGL3-basic vector. Mutation was confirmed using DNA sequencing.

Cells were transfected ${ }^{46,47}$ using Fugene (Promega) according to manufacturer's instructions. Renilla reniformis luciferase expression vector pRL-Tk (Promega) was used for transfection efficiency. The luciferase activity was measured using Dual Luciferase Assay System (Promega). For immunoblotting assays, cells were transfected with appropriate amounts of various plasmids, as indicated in figure legends.

RNA extraction and reverse transcription-PCR assays. Total RNA was isolated, reverse transcribed and amplified by PCR as described ${ }^{48}$ using specific primers (listed in Supplementary Table S1).

Immunoblotting analysis. Cells were lysed for protein extraction, and total or cytosolic/nuclear extracts were prepared and subjected to SDSPAGE as described. ${ }^{34,49}$

Immunofluorescence. Immunofluorescence analysis was performed as previously described, with minor modifications. ${ }^{50}$ Briefly, cells were fixed with $3.4 \%$ paraformaldehyde, permeabilized with $0.2 \%$ Triton $\mathrm{X}-100$, followed by blocking with BSA ( $3 \%, 30 \mathrm{~min})$, and incubated with anti-DAX-1 antibody $\left(4^{\circ} \mathrm{C}\right.$, overnight) and with fluorescein-conjugated secondary antibody ( $30 \mathrm{~min}$, room temperature). IgG primary antibody is used as negative control. DAPI staining is used for nuclei detection. Fluorescence was photographed with OLYMPUS BX51 microscope, $\times 100$ objective.

DNA affinity precipitation assay. DAPA was performed as described,$^{51}$ using the biotinylated sense oligonucleotides listed in Supplementary Table S2.

Electrophoretic mobility shift assay. Nuclear protein extracts were prepared and EMSA was performed as previously described. ${ }^{52}$ The oligonucleotides used as probes and as cold competitors (Sigma-Aldrich, Italy) are listed in Supplementary Table S2. Anti-AR antibody or normal IgG were included in some of the reaction mixtures.

Chromatin immunoprecipitation. ChIP assay was performed as described. ${ }^{53}$ Immunocleared chromatin was precipitated with anti-AR, -RNA Polymerase II, -DAX-1 and -N-CoR antibodies. Normal rabbit or mouse lgGs are as negative controls. Immunoprecipitated DNA was analyzed by RT-PCR using the specific primers listed in Supplementary Table S1.

RNA silencing. AR gene silencing experiments were performed as described..$^{54}$ For DAX-1 gene silencing experiments, custom synthesized siRNA (Ambion) annealed duplexes were used for effective depletion of DAX-1 mRNA. Scrambled siRNA is used as a control for non-sequence-specific effects. Cells were transfected with $80 \mathrm{pmol}$ of siDAX-1 or Scramble siRNA, using Lipofectamine2000 (Invitrogen Life Technologies), following the manufacturer's instructions.

Statistical analysis. All data were expressed as the mean \pm S.D. of three independent experiments. Statistical significances were tested using Student's t-test.

\section{Conflict of Interest}

The authors declare no conflict of interest.
Acknowledgements. The authors thank Dr. E.R. Simpson, Dr. C.D. Clyne and Dr. M.J. McPhaul for generously providing P450 aromatase promoter plasmids. This work was supported by Progetti di Ricerca di Interesse Nazionale (PRIN)Ministero Istruzione Università e Ricerca (MIUR) [grant number 20085Y7XT5]; and Associazione Italiana Ricerca sul Cancro (AIRC) [grant number 11595]. Reintegration AIRC/Marie Curie International Fellowship in Cancer Research to I. Barone.

1. Chen GG, Zeng Q, Tse GM. Estrogen and its receptors in cancer. Med Res Rev 2008; 28: 954-974.

2. Fowler KA, Gill K, Kirma N, Dillehay DL, Tekmal RR. Overexpression of aromatase leads to development of testicular leydig cell tumors: an in vivo model for hormone-mediated TesticularCancer. Am J Pathol 2000; 156: 347-353.

3. Yager JD, Davidson NE. Estrogen carcinogenesis in breast cancer. N Engl J Med 2006; 354: $270-282$.

4. Jemal A, Siegel R, Ward E, Hao Y, Xu J, Thun MJ. Cancer statistics 2009. CA Cancer $J$ Clin 2009; 59: 225-249.

5. Sasano H, Miki Y, Nagasaki S, Suzuki T. In situ estrogen production and its regulation in human breast carcinoma: from endocrinology to intracrinology. Pathol Int 2009; 59: $777-789$

6. Forbes JF, Cuzick J, Buzdar A, Howell A, Tobias JS, Baum M. Effect of anastrozole and tamoxifen as adjuvant treatment for early-stage breast cancer: 100-month analysis of the ATAC trial. Lancet Oncol 2008; 9: 45-53.

7. Park S, Koo J, Park HS, Kim JH, Choi SY, Lee JH et al. Expression of androgen receptors in primary breast cancer. Ann Oncol 2010; 21: 488-492.

8. Castellano I, Allia E, Accortanzo V, Vandone AM, Chiusa L, Arisio R et al. Androgen receptor expression is a significant prognostic factor in estrogen receptor positive breast cancers. Breast Cancer Res Treat 2010; 124: 607-617.

9. Yu Q, Niu Y, Liu N, Zhang JZ, Liu TJ, Zhang RJ et al. Expression of androgen receptor in breast cancer and its significance as a prognostic factor. Ann Oncol 2011; 22: 1288-1294.

10. Peters AA, Buchanan G, Ricciardelli C, Bianco-Miotto T, Centenera MM, Harris JM et al. Androgen receptor inhibits estrogen receptor-alpha activity and is prognostic in breast cancer. Cancer Res 2009; 69: 6131-6140.

11. Berns EM, Dirkzwager-Kiel MJ, Kuenen-Boumeester V, Timmermans M, Verhoog LC, van den Ouweland AM et al. Androgen pathway dysregulation in BRCA1-mutated breast tumors. Breast Cancer Res Treat 2003; 79: 121-127.

12. Lin Fde M, Pincerato KM, Bacchi CE, Baracat EC, Carvalho FM. Coordinated expression of oestrogen and androgen receptors in HER2-positive breast carcinomas: impact on proliferative activity. J Clin Pathol 2012; 65: 64-68.

13. Lanzino M, De Amicis F, McPhaul MJ, Marsico S, Panno ML, Ando S. Endogenous coactivator ARA70 interacts with estrogen receptor alpha (ERalpha) and modulates the functional ERalpha/androgen receptor interplay in MCF-7 cells. J Biol Chem 2005; 280: 20421-20430

14. Ando S, De Amicis F, Rago V, Carpino A, Maggiolini M, Panno ML et al. Breast cancer: from estrogen to androgen receptor. Mol Cell Endocrinol 2002; 193: 121-128.

15. Greeve MA, Allan RK, Harvey JM, Bentel JM. Inhibition of MCF-7 breast cancer cell proliferation by 5alpha-dihydrotestosterone; a role for p21(Cip1/Waf1). J Mol Endocrinol 2004; 32: 793-810.

16. Lanzino M, Sisci D, Morelli C, Garofalo C, Catalano S, Casaburi I et al. Inhibition of cyclin D1 expression by androgen receptor in breast cancer cells-identification of a novel androgen response element. Nucleic Acids Res 2010; 38: 5351-5365.

17. Bardoni B, Zanaria E, Guioli S, Floridia G, Worley KC, Tonini G et al. A dosage sensitive locus at chromosome Xp21 is involved in male to female sex reversal. Nat Genet 1994; 7 : 497-501.

18. Zanaria E, Muscatelli F, Bardoni B, Strom TM, Guioli S, Guo W et al. An unusual member of the nuclear hormone receptor superfamily responsible for $X$-linked adrenal hypoplasia congenita. Nature 1994; 372: 635-641.

19. Zazopoulos E, Lalli E, Stocco DM, Sassone-Corsi P. DNA binding and transcriptional repression by DAX-1 blocks steroidogenesis. Nature 1997; 390: 311-315.

20. Guo W, Burris TP, McCabe ER. Expression of DAX-1, the gene responsible for X-linked adrenal hypoplasia congenita and hypogonadotropic hypogonadism, in the hypothalamicpituitary-adrenal/gonadal axis. Biochem Mol Med 1995; 56: 8-13.

21. Lalli E, Sassone-Corsi P. DAX-1, an unusual orphan receptor at the crossroads of steroidogenic function and sexual differentiation. Mol Endocrinol 2003; 17: 1445-1453.

22. Crawford PA, Dorn C, Sadovsky Y, Milbrandt J. Nuclear receptor DAX-1 recruits nuclear receptor corepressor N-CoR to steroidogenic factor 1. Mol Cell Biol 1998; 18: 2949-2956.

23. Hanley NA, Rainey WE, Wilson DI, Ball SG, Parker KL. Expression profiles of SF-1, DAX1, and CYP17 in the human fetal adrenal gland: potential interactions in gene regulation. Mol Endocrinol 2001; 15: 57-68.

24. Wang ZJ, Jeffs B, Ito M, Achermann JC, Yu RN, Hales DB et al. Aromatase (Cyp19) expression is up-regulated by targeted disruption of Dax1. Proc Natl Acad Sci USA 2001; 98: 7988-7993.

25. Reincke M, Beuschlein F, Lalli E, Arlt W, Vay S, Sassone-Corsi P et al. DAX-1 expression in human adrenocortical neoplasms: implications for steroidogenesis. J Clin Endocrinol Metab 1998; 83: 2597-2600. 
26. Chae BJ, Lee A, Bae JS, Song BJ, Jung SS. Expression of nuclear receptor DAX-1 and androgen receptor in human breast cancer. J Surg Oncol 2011; 103: 768-772.

27. Conde I, Alfaro JM, Fraile B, Ruiz A, Paniagua R, Arenas MI. DAX-1 expression in human breast cancer: comparison with estrogen receptors ER-alpha, ER-beta and androgen receptor status. Breast Cancer Res 2004; 6: R140-R148.

28. Park YY, Ahn SW, Kim HJ, Kim JM, Lee IK, Kang H et al. An autoregulatory loop controlling orphan nuclear receptor DAX-1 gene expression by orphan nuclear receptor ERRgamma. Nucleic Acids Res 2005; 33: 6756-6768.

29. Abd-Elaziz M, Akahira J, Moriya T, Suzuki T, Yaegashi N, Sasano H. Nuclear receptor DAX-1 in human common epithelial ovarian carcinoma: an independent prognostic factor of clinical outcome. Cancer Sci 2003; 94: 980-985.

30. Saito S, Ito K, Suzuki T, Utsunomiya H, Akahira J, Sugihashi $Y$ et al. Orphan nuclear receptor DAX-1 in human endometrium and its disorders. Cancer Sci 2005; 96: $645-652$.

31. Nakamura $Y$, Suzuki T, Arai $Y$, Sasano H. Nuclear receptor DAX1 in human prostate cancer: a novel independent biological modulator. Endocr J 2009; 56: 39-44.

32. Zoppi S, Marcelli M, Deslypere JP, Griffin JE, Wilson JD, McPhaul MJ. Amino acid substitutions in the DNA-binding domain of the human androgen receptor are a frequent cause of receptor-binding positive androgen resistance. Mol Endocrinol 1992; 6 : 409-415.

33. Verrijdt G, Haelens A, Claessens F. Selective DNA recognition by the androgen receptor as a mechanism for hormone-specific regulation of gene expression. Mol Genet Metab 2003 78: $175-185$.

34. Sirianni R, Chimento A, Malivindi R, Mazzitelli I, Ando S, Pezzi V. Insulin-like growth factor-I, regulating aromatase expression through steroidogenic factor 1 , supports estrogendependent tumor Leydig cell proliferation. Cancer Res 2007; 67: 8368-8377.

35. Sasano H, Harada N. Intratumoral aromatase in human breast, endometrial, and ovarian malignancies. Endocr Rev 1998; 19: 593-607.

36. Barone I, Giordano C, Malivindi R, Lanzino M, Rizza P, Casaburi I et al. Estrogens and PTP1B function in a novel pathway to regulate aromatase enzymatic activity in breast cancer cells. Endocrinology 2012; 153: 5157-5166.

37. Clipsham R, McCabe ER. DAX1 and its network partners: exploring complexity in development. Mol Genet Metab 2003; 80: 81-120.

38. Ikeda Y, Swain A, Weber TJ, Hentges KE, Zanaria E, Lalli E et al. Steroidogenic factor 1 and Dax-1 colocalize in multiple cell lineages: potential links in endocrine development. Mol Endocrinol 1996; 10: 1261-1272.

39. Lalli E, Melner MH, Stocco DM, Sassone-Corsi P. DAX-1 blocks steroid production at multiple levels. Endocrinology 1998; 139: 4237-4243.

40. Zhang H, Slewa A, Janssen E, Skaland I, Yu Y, Gudlaugsson E et al. The prognostic value of the orphan nuclear receptor DAX-1 (NROB1) in node-negative breast cancer. Anticancer Res 2011; 31: 443-449.

41. Tamai KT, Monaco L, Alastalo TP, Lalli E, Parvinen M, Sassone-Corsi P. Hormonal and developmental regulation of DAX-1 expression in Sertoli cells. Mol Endocrinol 1996; 10: 1561-1569.

42. Suzuki T, Miki Y, Moriya T, Akahira J, Ishida T, Hirakawa $\mathrm{H}$ et al. 5Alpha-reductase type 1 and aromatase in breast carcinoma as regulators of in situ androgen production. Int J Cancer 2007; 120: 285-291.
43. Takagi K, Miki Y, Nagasaki S, Hirakawa H, Onodera $Y$, Akahira J et al. Increased intratumoral androgens in human breast carcinoma following aromatase inhibitor exemestane treatment. Endocr Relat Cancer 2010; 17: 415-430.

44. Rovito D, Giordano C, Vizza D, Plastina P, Barone I, Casaburi I et al. Omega-3 PUFA ethanolamides DHEA and EPEA induce autophagy through PPAR $\gamma$ activation in MCF-7 breast cancer cells. J Cell Physiol 2013; 228: 1314-1322.

45. Catalano S, Panza S, Malivindi R, Giordano C, Barone I, Bossi G et al. Inhibition of Leydig tumor growth by farnesoid $X$ receptor activation: the in vitro and in vivo basis for a novel therapeutic strategy. Int J Cancer 2013; 132: 2237-2247.

46. Recchia AG, Musti AM, Lanzino M, Panno ML, Turano E, Zumpano R et al. A cross-talk between the androgen receptor and the epidermal growth factor receptor leads to p38 MAPK-dependent activation of mTOR and cyclinD1 expression in prostate and lung cancer cells. Int J Biochem Cell Biol 2009; 41: 603-614.

47. Guido C, Panza S, Santoro M, Avena P, Panno ML, Perrotta I et al. Estrogen receptor beta $(E R \beta)$ produces autophagy and necroptosis in human seminoma cell line through the binding of the Sp1 on the phosphatase and tensin homolog deleted from chromosome 10 (PTEN) promoter gene. Cell Cycle 2012; 11: 2911-2921.

48. Lanzino M, Garofalo C, Morelli C, Le Pera M, Casaburi I, McPhaul MJ et al. Insulin receptor substrate 1 modulates the transcriptional activity and the stability of androgen receptor in breast cancer cells. Breast Cancer Res Treat 2009; 115: 297-306.

49. Chimento A, Sirianni R, Zolea F, De Luca A, Lanzino M, Catalano S et al. Nandrolone and stanozolol induce Leydig cell tumor proliferation through an estrogen-dependent mechanism involving IGF-I system. J Cell Physiol 2012; 227: 2079-2088.

50. Barone I, Catalano S, Gelsomino L, Marsico S, Giordano C, Panza S et al. Leptin mediates tumor-stromal interactions that promote the invasive growth of breast cancer cells. Cancer Res 2012; 72: 1416-1427.

51. Morelli C, Lanzino M, Garofalo C, Maris P, Brunelli E, Casaburi I et al. Akt2 inhibition enables the forkhead transcription factor $\mathrm{FoxO} a$ to have a repressive role in estrogen receptor alpha transcriptional activity in breast cancer cells. Mol Cell Biol 2010; 30: 857-870.

52. Giordano C, Vizza D, Panza S, Barone I, Bonofiglio D, Lanzino M et al. Leptin increases HER2 protein levels through a STAT3-mediated up-regulation of Hsp90 in breast cancer cells. Mol Oncol 2012; pii: S1574-7891: 00123-00128.

53. Catalano S, Rizza P, Gu G, Barone I, Giordano C, Marsico S et al. Fas ligand expression in TM4 Sertoli cells is enhanced by estradiol 'in situ' production. J Cell Physiol 2007; 211: 448-456.

54. Casaburi I, Avena P, Lanzino M, Sisci D, Giordano F, Maris P et al. Chenodeoxycholic acid through a TGR5-dependent CREB signaling activation enhances cyclin D1 expression and promotes human endometrial cancer cell proliferation. Cell Cycle 2012; 11: 2699-2710.

(c) $(-)$ Cell Death and Disease is an open-access journal published by Nature Publishing Group. This work is licensed under a Creative Commons Attribution-NonCommercialNoDerivs 3.0 Unported License. To view a copy of this license, visit http://creativecommons.org/licenses/by-nc-nd/3.0/

\section{Supplementary Information accompanies this paper on Cell Death and Disease website (http://www.nature.com/cddis)}

